\title{
Recent results of strong interaction program from NA61/SHINE experiment at CERN SPS
}

\author{
Georgy Melkumov ${ }^{1, *}$ for the NA61/SHINE Collaboration \\ ${ }^{1}$ Joint Institute for Nuclear Research, 141980 Dubna, Russia
}

\begin{abstract}
.
The NA61/SHINE experiment at the CERN SPS pursues a rich program of strong interactions. The main physics goals of this program are the study of properties of the onset of deconfinement and search for the signatures of the critical point of strongly interacting matter by performing the two-dimensional scan in a broad region of energy (beam momentum 13A-158A GeV/c) and system size ( $\mathrm{p}+\mathrm{p}, \mathrm{Be}+\mathrm{Be}, \mathrm{Ar}+\mathrm{Sc}$ and $\mathrm{Xe}+\mathrm{La})$.

Recent NA61/SHINE results on particle spectra and event-by-event fluctuations in $\mathrm{p}+\mathrm{p}, \mathrm{Be}+\mathrm{Be}$ and $\mathrm{Ar}+\mathrm{Sc}$ collisions are shown with emphasis on the measurements of particle ratios, namely of pion a strangeness production, multiplicity fluctuations versus energy and the system size of colliding nuclei.

It will be shown that the hadron production properties in heavy ion collisions which change rapidly in the low SPS energy domain and are interpreted as the beginning of quark-gluon plasma production - onset of deconfinement could be also the case in inelastic $\mathrm{p}+\mathrm{p}$ interactions and probably in $\mathrm{Be}+\mathrm{Be}$ collisions.

The paper presents a selection of NA61/SHINE results on particle production properties discussed together with existing data from the NA49 collaboration. The evolution of non-monotonic structures in the pion and strangeness production as a function of the system size and energy is addressed. The rapid change of hadron production properties from $\mathrm{p}+\mathrm{p}$ and $\mathrm{Be}+\mathrm{Be}$ up to $\mathrm{Ar}+\mathrm{Sc}$ and $\mathrm{Pb}+\mathrm{Pb}$ collisions can be interpreted as the beginning of the large clusters formation of strongly interacting matter - the onset of fireball.

The NA61/SHINE strong interaction programme is presented including the recent status on proton intermittency analysis and strongly intensive fluctuation observables of particle multiplicity and transverse momentum.
\end{abstract}

\section{Introduction}

Exploration of predicted prominent features of the phase diagram of strongly interacting matter, particularly the deconfinement phase transition from hadrons to a system of quasi-free quarks and gluons (QGP) and quest for the critical point is the primary goal of a number of contemporary experiments, developing projects and numerous theoretical investigations.

The main goal of the strong interaction program NA61/SHINE is to search for the critical point of strongly interacting matter (CP) and study the properties of the onset of deconfinemet. The program was motivated by the discovery of the onset of deconfinement in the

\footnotetext{
*e-mail: Georgui.Melkoumov@ cern.ch
} 
central $\mathrm{Pb}+\mathrm{Pb}$ collision at the low energy range of SPS provided by the NA49 collaboration. A tantalizing hint on the CP indication has been observed by NA49 experiment for the medium-size nuclei at the top SPS energy. It also motivated the physics program of strongly interacting matter.

The NA61/SHINE Collaboration at CERN SPS pursues this challenging program performing a two-dimensional scan in a broad region of the QCD phase diagram by varying the momentum (13A-158A GeV/c) and the size of colliding systems (p+p, Be+Be, Ar+Sc, $\mathrm{Xe}+\mathrm{La}$ and $\mathrm{Pb}+\mathrm{Pb})$.

The NA61/SHINE experiment [1] is a multi-purpose fixed target experiment at the Super Proton Synchrotron (SPS) of the European Organization for Nuclear Research (CERN). The strong interactions programme of NA61/SHINE is devoted to the studies of the Quantum Chromodynamics phase structure and, in particular, search for the critical point (CP) [2] of strongly interacting matter. It is expected that there will be a non-monotonic dependence of hadron production properties, in particular fluctuations of a number of observables on the energy and system size in this scan due to the phase transition of strongly interacting matter and possible existence of the CP [3]. Some possible indication of this behaviour has already been observed by the NA49 experiment [4]

\section{Onset of deconfinement in hadron production}

There is a firm notion that in central collisions of heavy nuclei at the top SPS energy $\left(\sqrt{s_{N N}}\right.$ $=17.3 \mathrm{GeV})$ as well as at RHIC energies $\left(\sqrt{s_{N N}}=130\right.$ and $\left.200 \mathrm{GeV}\right)$ a transient state of deconfined quarks and gluons (QGP) is produced. It is argued by the observation of the signatures predicted for the QGP.

The energy dependence of the hadron production properties has been studied to find out whether the early stage fireball actually reaches hadron deconfinement. This program was originally motivated by the predictions of the Statistical Model of Early Stage (SMES) [5] assuming that the energy threshold for deconfinement is located at low SPS energies.
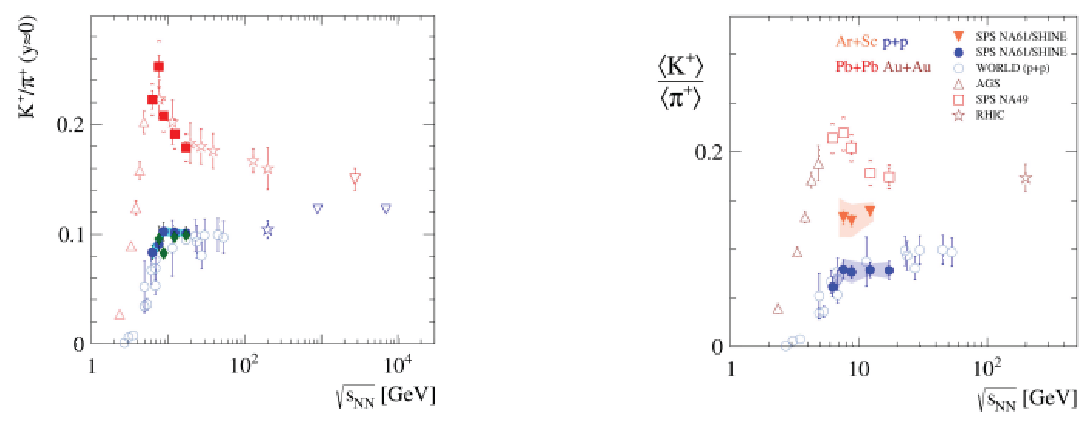

Figure 1. Energy dependence of the $K^{+} / \pi^{+}$ratio at midrapidity (left) and the $\left\langle K^{+}\right\rangle /\left\langle\pi^{+}\right\rangle$ratio (right) for total particle multiplicity in $4 \pi$ in $\mathrm{Pb}+\mathrm{Pb}$ and $\mathrm{Au}+\mathrm{Au}$ (red), $\mathrm{Be}+\mathrm{Be}$ (green) and $\mathrm{p}+\mathrm{p}$ (blue) reactions. The closed symbols mark NA49 and NA61/SHINE data while the open symbols indicate all other 'world' data

Several structures in excitation functions were expected within the SMES: a "kink" in the increase of the pion yield per participant nucleon (change of slope due to increased entropy as a consequence of the activation of partonic degrees of freedom), a sharp peak "horn" in the 
strangeness to entropy ratio, and a "step" in the inverse slope parameter of transverse mass spectra (constant temperature and pressure in a mixed phase). These signatures were indeed observed in A+A collisions by the NA49, thus locating the onset of deconfinement at the energy around $30 \mathrm{~A} \mathrm{GeV}\left(\sqrt{s_{N N}}=7.6 \mathrm{GeV}\right)[6,7]$.

Rapid changes of the ratios $K^{+} / \pi^{+}$at mid-rapidity and $\left\langle K^{+}\right\rangle /\left\langle\pi^{+}\right\rangle$as function of collision energy "horn" were observed in $\mathrm{Pb}+\mathrm{Pb}$ collisions by NA49 experiment. They were predicted by SMES model as a signature of the onset of decomfinement. These two ratios together with new NA61/SHINE results [8] for $\mathrm{Be}+\mathrm{Be}$ and $\mathrm{Ar}+\mathrm{Sc}$ collisions are shown in Fig. 1. A plateau-like structure is visible in $\mathrm{p}+\mathrm{p}$ interactions. The ratio $K^{+} / \pi^{+}$at mid-rapidity from $\mathrm{Be}+\mathrm{Be}$ collisions is close to the $\mathrm{p}+\mathrm{p}$ points.

Originally a smooth energy dependence of $\mathrm{K}^{+} / \pi^{+}$ratio for $\mathrm{p}+\mathrm{p}$ reactions was assumed. The new precise data by NA61/SHINE do not exclude such a trend within error bars, however it can also indicate a step-like behavior as a reduced 'shadow' of the "horn" structure seen in $\mathrm{Pb}+\mathrm{Pb}$ reactions. It could hint on some possible transition from a confined system as it is the case in A+A collisions.

Charge kaon spectra and yields for $\mathrm{Ar}+\mathrm{Sc}$ interactions have been measured at beam momenta of $30 \mathrm{~A}, 40 \mathrm{~A}$ and $75 \mathrm{~A} \mathrm{GeV} / \mathrm{c}$. The double differential $p_{T}$-extrapolated and rapidity integrated spectra were used to derived mean multiplicity as shown in Fig. 1(right). The ratio $\left\langle K^{+}\right\rangle /\left\langle\pi^{+}\right\rangle$in $\mathrm{Ar}+\mathrm{Sc}$ collisions is between the ratio in inelastic $\mathrm{p}+\mathrm{p}$ interactions and the one in violent $\mathrm{Pb}+\mathrm{Pb}$ collisions. It shows a similar dependence on the collision energy as in $\mathrm{p}+\mathrm{p}$ interactions.
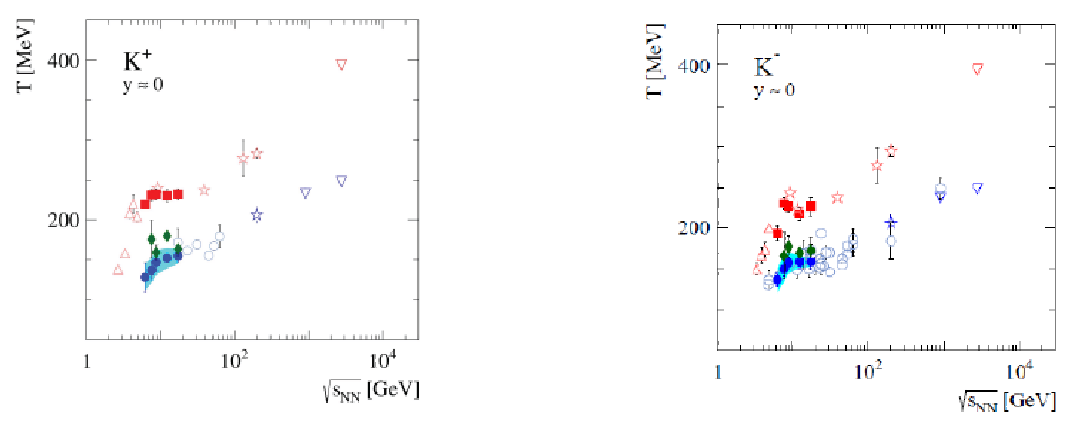

Figure 2. Inverse slope parameter of transverse mass distributions for $K^{+}$and $K^{-}$as a function of the collision energy. The closed symbols mark NA49 and NA61/SHINE data while the open symbols indicate all other 'world' data

Fig. 2 shows the energy dependence of the inverse slope parameter of transverse mass distributions for $K^{+}$and $K^{-}$. The Statistical Model of Early Stage (SMES) predicts the first order phase transition to the QGP between the top AGS and top SPS energies. In the transition region the constant temperature and pressure in the mixed phase and the increase of the number of internal degrees of freedom are predicted. A plateau "step" in the energy dependence of the inverse slope $T$ was observed by the NA49 experiment in $\mathrm{Pb}+\mathrm{Pb}$ collision for $m_{T}$ spectra of $K^{ \pm}$. It was expected for the onset of deconfinement due to the presence of a mixed phase of hadron gas (HRG) and quark-gluon plasma(QGP).

In $\mathrm{p}+\mathrm{p}$ interactions at SPS energies the inverse slope parameter $T$ of $m_{T}$-spectra shows a qualitatively similar structure in central $\mathrm{Pb}+\mathrm{Pb}$ collisions "step" and this behavior seems to emerge also in $\mathrm{p}+\mathrm{p}$ and $\mathrm{Be}+\mathrm{Be}$ reactions, as visible in Fig. 2 . The value of $T$ parameter in $\mathrm{Be}+\mathrm{Be}$ collisions is slightly above those in $\mathrm{p}+\mathrm{p}$ interactions. 
Thus, the new precise data of NA61/SHINE reveal the energy dependence of particle production properties in $\mathrm{p}+\mathrm{p}$ and $\mathrm{Be}+\mathrm{Be}$ interactions that exhibit some features qualitatively similar to those attributed to deconfined matter in the central collisions of heavy nuclei.

\section{Onset of deconfinement and Onset of fireball}

Fig. 3 shows the system size dependence of the ratio $K^{+}$and $\pi^{+}$yields at midrapidity at $30 \mathrm{~A}$ $\mathrm{GeV} / \mathrm{c}$ and $150 \mathrm{~A} \mathrm{GeV} / \mathrm{c}$. A rapid increase of the particle ratio when moving from $\mathrm{Be}+\mathrm{Be}$ to $\mathrm{Ar}+\mathrm{Sc}$ collisions is observed, while the $\mathrm{Be}+\mathrm{Be}$ results are close to $\mathrm{p}+\mathrm{p}$ independently of the collision energy. Moreover generally, the data show a jump between light $(\mathrm{p}+\mathrm{p}, \mathrm{Be}+\mathrm{Be})$ and intermediate/heavy $(\mathrm{Ar}+\mathrm{Sc}, \mathrm{Pb}+\mathrm{Pb})$ systems. The additional NA49 data points are invoked to exhibit the trend of the size dependence some more in detail shown in Fig. 4(left).
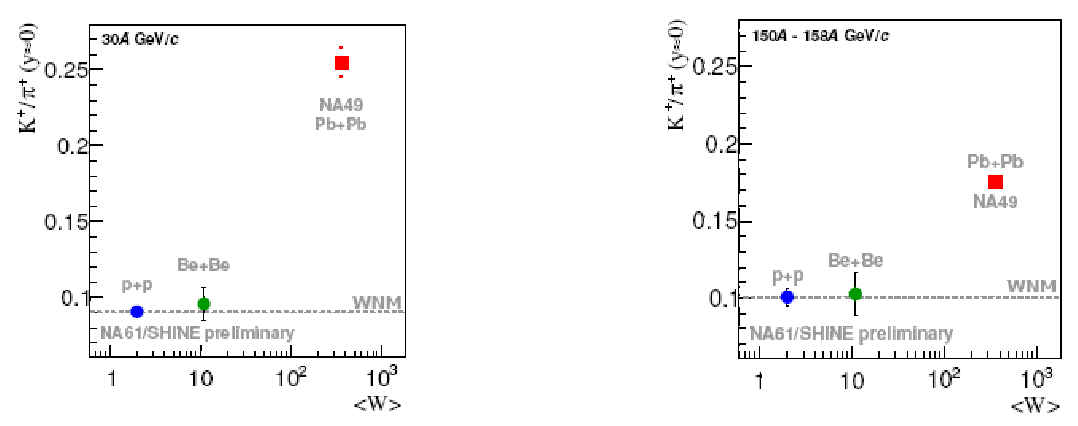

Figure 3. Ratio of the $K^{+} / \pi^{+}$particle yields at mid-rapidity in $\mathrm{p}+\mathrm{p}, \mathrm{Be}+\mathrm{Be}$ and $\mathrm{Ar}+\mathrm{Sc}$ interactions at the beam momenta of $30 \mathrm{~A} \mathrm{GeV} / \mathrm{c}$ (left) and $150 \mathrm{~A} / 158 \mathrm{~A} \mathrm{GeV} / \mathrm{c}$ (right) as a function of the mean number of wounded nucleons (number of participating nucleons)

The preliminary $\mathrm{Be}+\mathrm{Be}$ points remain close to $\mathrm{p}+\mathrm{p}$ data that seems to be consistent with the Wounded Nucleon Model (WNM) [9] (Fig. 3) rather than the statistical model predictions. The $K^{+} / \pi^{+}$ratio in $\mathrm{p}+\mathrm{p}$ interactions is below the predictions of statistical models.

However, the ratio in central $\mathrm{Pb}+\mathrm{Pb}$ collisions is close to statistical model predictions for large volume systems [10]. This qualitatively goes along with the weakening of "canonical suppression" of the mean multiplicity [11] with the increasing cluster size predicted in the framework of statistical SM(IB-CE) and SM(IB-GCE) models. The known prediction of the Statistical Model with Ideal Boltzmann Gas within Canonical Ensemble, SM(IB-CE), and Grand Canonical Ensemble, SM(IB-GCE), is the "canonical suppression" of the mean multiplicity [11] within GCE to be proportional to the cluster volume.

The above qualitative expectation has been confronted with the NA49 and NA61/SHINE data [12] which show the rapid change of the $K^{+} / \pi^{+}$ratio at midrapidity (Fig. 4(left)). One suggests that there are two regimes: Wounded Nucleon Model regime for light systems with incoherent superposition of particles from wounded nucleons (independently interacting nucleons) and Statistical Model regime for heavier systems with large clusters. Thus the observed rapid change of hadron production properties which starts when moving from $\mathrm{Be}+\mathrm{Be}$ to $\mathrm{Ar}+\mathrm{Sc}$ collisions, can be interpreted as the beginning of large clusters production of strongly interacting matter - the onset of fireball [12]. The onset of fireball would mark the transition from small "non-statistical" systems into the region of large clusters of strongly interacting matter which can be described by statistical models. 
Taking into account the onset of deconfinemet that is well defined in central $\mathrm{Pb}+\mathrm{Pb}$ collisions (although its presence in the light systems also, in particular, inelastic $\mathrm{p}+\mathrm{p}$ interactions is questionable) one can suggest the following. The two-dimensional scan performed by NA61/SHINE by means of varying the collision energy and the nuclear mass number of colliding nuclei indicates four domains of hadron production properties separated by two thresholds: the onset of deconfinement and the onset of fireball. The sketch presented in Fig. 4(right) illustrates this conclusion.
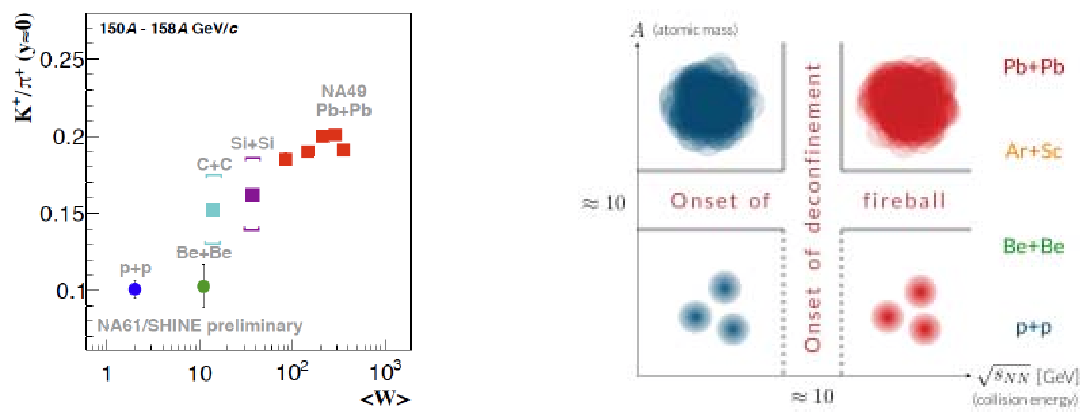

Figure 4. (left) System size dependence of $K^{+} / \pi^{+}$ratio at mid-rapidity for $\mathrm{p}+\mathrm{p}, \mathrm{Be}+\mathrm{Be}$ (NA61/SHINE) and $\mathrm{C}+\mathrm{C}, \mathrm{Si}+\mathrm{Si}, \mathrm{Pb}+\mathrm{Pb}(\mathrm{NA} 49)$ collisions at $150 \mathrm{~A} / 158 \mathrm{~A} \mathrm{GeV} / \mathrm{c}$. (right) Twodimensional sketch of four domains of the hadron production properties separated by two thresholds - the onset of deconfinements and the onset of fireball, as indicated by the NA61/SHINE scan of the phase diagram over the energy and size of the colliding systems

\section{Critical point and critical fluctuations}

One of the primary goals of the NA61/SHINE scanning program is to find the evidence for the QCD critical point looking for a maximum of the event-by-event fluctuations.

A characteristic feature of a second order phase transition at the critical point is the divergence of the correlation length. The system becomes scale invariant. This leads to large fluctuations in particle multiplicity and other characteristics of events. Moreover, these fluctuations have specific characteristics [13]. Also other properties of the system should be sensitive to the vicinity of the critical point [3]. Thus, when scanning the phase diagram a region of increased fluctuations may signal the evidence of the critical point and it is expected to look as a "hill" of fluctuations (Fig. 5(left)).

In the experimental search for the critical point one should use proper measures of fluctuations especially strongly intensive measures which are independent of the volume and its fluctuations while being not sensitive to material conservation laws.

The NA61/SHINE uses the strongly intensive measures $\Delta\left[P_{T}, N\right]$ and $\Sigma\left[P_{T}, N\right]$ to study transverse momentum and multiplicity fluctuations [14] in which $P_{T}$ and $N$ are sum of the absolute values of particle transverse momenta and particle multiplicity, respectively. They depend neither on the number of wounded nucleons $\langle W\rangle$ nor on fluctuations of W. They also do not depend on volume and volume fluctuations. These fluctuation measures $\Delta\left[P_{T}, N\right]$ and $\Sigma\left[P_{T}, N\right]$ have two reference values: they are equal to zero in case of no fluctuations and one in case of independent particle production. No indication for the critical point has been 

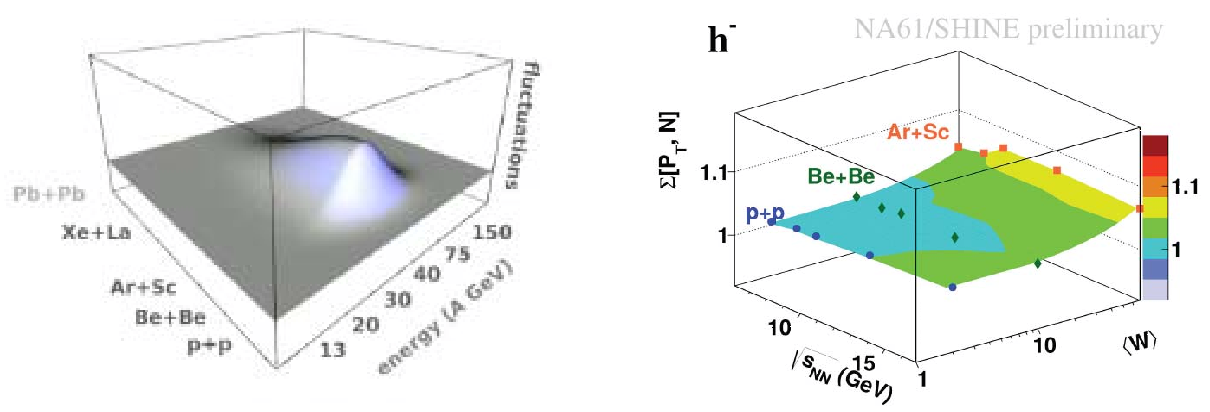

Figure 5. (left) Sketch of the "hill" of fluctuations which may be observed in the (beam momentum) vs. (system size) scan of NA61/SHINE provided the freeze-out parameters $\left(T-\mu_{B}\right)$ of the system produced in the collision are close to the critical point. (right) $\Sigma\left[P_{T}, N\right]$ measured by NA61/SHINE in inelastic $\mathrm{p}+\mathrm{p}$ interactions and violent $\mathrm{Be}+\mathrm{Be}$ and $\mathrm{Ar}+\mathrm{Sc}$ collisions at the CERN SPS energies. Results rerefer to negatively charged hadrons at forward rapidity $\left(0<y_{\pi}<y_{\text {beam }}\right)$ and $p_{T}<1.5 \mathrm{GeV} / \mathrm{c}$
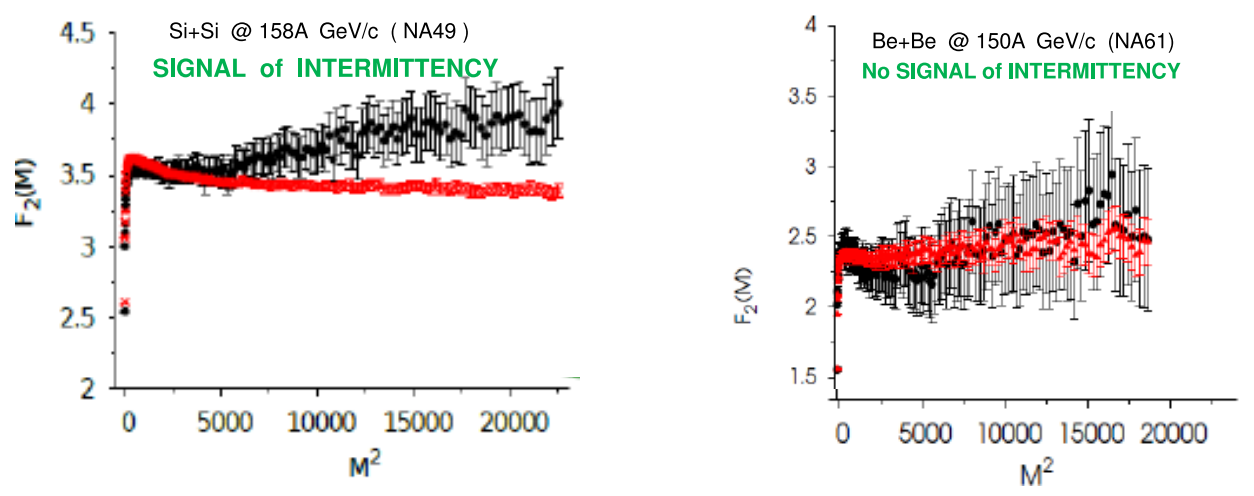

Figure 6. (left) Scaled factorial moment $F_{2}(M)$ of identified protons for central $\mathrm{Si}+\mathrm{Si}$ collision system at $158 \mathrm{~A} \mathrm{GeV} / \mathrm{c}$ for data (black points) and event-mixed non-critical background (red points). There is an obvious difference seen between the data and background. Analysis of the background subtracted data reveals a power low behavior for factorial moment $F_{2}(M)$ that is predicted for the system at the vicinity to the critical point. (right) Similar analysis for central $\mathrm{Be}+\mathrm{Be}$ reactions indicate no visible intermittency effect, namely the data for $F_{2}(M)$ are fully reproduced by the mixed events

observed so far in this analysis [15]. This is evident in Fig 5 (right). The analysis is ongoing and the results are awaited to clarify the picture.

However, the previous intermittency analysis of NA49 has indicated a significant power low fluctuation of proton and pion-pion densities in $p_{T}$ space of $\mathrm{Si}+\mathrm{Si}$ collisions at $158 \mathrm{~A}$ $\mathrm{GeV} / \mathrm{c}$ with a power low exponent approaching to the theoretical predictions for the QCD critical point $[16,17]$, while no intermittency behavior was visible in $\mathrm{C}+\mathrm{C}$ and $\mathrm{Pb}+\mathrm{Pb}$ systems at the same energy. This strongly motivated similar analysis of the NA61/SHINE data which is underway now.

Potentially important is the analysis of proton intermittency. Following [18], for the collision system in vicinity of the critical point, the critical component of a second order factorial 
moments $F_{2}(M)$ of proton multiplicity in the cell $(M \times M)$ should scale like a power law with the number of cells $M^{2}[19,20]$.

Intermittency analysis was performed for NA61/SHINE data on $\mathrm{Be}+\mathrm{Be}$ collisions at $150 \mathrm{~A} \mathrm{GeV} / \mathrm{c}$ beam momentum. No visible intermittency effect has been observed. Namely, for central $\mathrm{Be}+\mathrm{Be}$ reactions at top SPS energy, the obtained values of $F_{2}(M)$ appear to be fully reproduced by the mixed events (background), indicating no signal of the intermittent component (Fig. 6(right)). For central $\mathrm{Si}+\mathrm{Si}$ collisions, on the other hand, a deviation from the event-mixed non-critical background has become evident in the above mentioned analysis of the NA49 data (Fig. 6(left)).

Finally, it should be emphasized that very preliminary recent results of the intermittency analysis in $\mathrm{Ar}+\mathrm{Sc}$ collisions from NA61/SHINE confirm the early $\mathrm{Si}+\mathrm{Si}$ result on possible indication of the critical point [21]. Thus, new experimental data from the NA61/SHINE two-dimensional scan, in particular, on the energy dependence of the intermittent signal, can answer the question on the presence of the critical point in the SPS energy regime.

\section{Summary}

A two-dimensional scan of the QCD phase diagram of strongly interacting matter has been conducted by NA61/SHINE in a wide region of the energy and the mass number of colliding nuclei. Comprehensive analysis of the data indicates four domains of hadron production properties separated by two threshold - the onset of deconfinement and the onset of fireball.

The energy dependence of particle production properties such as the particle ratios and transverse mass spectra in $\mathrm{p}+\mathrm{p}$ and $\mathrm{Be}+\mathrm{Be}$ interactions exhibits some features qualitatively similar to those attributed to the deconfined matter in the central collisions of heavy nuclei.

Several studies of fluctuations made by means of strongly intensive quantities have brought no clear fluctuation signal attributed to the critical point so far. However, the relevant indication is expected to be feasible for, at least, the Ar+Sc system at $150 \mathrm{~A} \mathrm{GeV}$ as suggested by the NA49 results for the $\mathrm{Si}+\mathrm{Si}$ system at $158 \mathrm{~A} \mathrm{GeV}$. Moreover, it should be stressed that very preliminary results of the proton intermittency in local fluctuations of baryon density at $\mathrm{Ar}+\mathrm{Sc}$ collisions from NA61/SHINE confirm the early $\mathrm{Si}+\mathrm{Si}$ result on possible indication of the critical point.

The future NA61/SHINE program on strong interactions is associated with the measurements of charm hadrons, mostly $\mathrm{D}$ mesons, produced in $\mathrm{Pb}+\mathrm{Pb}$ collisions at SPS energies.

\section{References}

[1] N. Abgral et al. (NA61 Collaboration), JINST 9, P06005 (2014)

[2] Z. Fodor and S. Katz, JHEP 04, 050 (2004)

[3] M. A. Stephanov, K. Rajagopal, E. V. Shuryak, Phys. Rev. D 60, 114028 (1999)

[4] K. Grebieszkov (NA49 Collababoration), Nucl. Phys. A 830, 575C (2009)

[5] M. Gazdzicki and M.I. Gorenstein, Acta. Phys. Polon. B 30, 2705 (1999)

[6] S. Afanisiev et al. (NA49 Collaboration), Phys. Rev. C 66, 054902 (2002)

[7] C. Alt et al. (NA49 Collaboration), Phys. Rev. C 77, 024903 (2008)

[8] S. Pulawski (NA61 Collaboration), PoS CPOD 2017, 010 (2018)

[9] A. Bialas, M. Bleszynski and W. Czyz, Nucl. Phys. B 111, 461 (1999)

[10] V. Becattini, J. Manninen and M. Gazdzicki, Phys. Rev. C 73, 044905 (2006)

[11] J. Rafelski and M. Danos, Phys. Lett. B 97, 279 (1980)

[12] M. Gazdzicki (NA61 Collaboration), PoS CPOD 2017, 012 (2018) 
[13] A. Bialas and R. C. Hwa, Phys. Lett. B 253, 436 (1991)

[14] A. Aduszkiewicz et al. (NA61 Collaboration), Eur. Phys. J. C 76(11), 635 (2016)

[15] A. Aduszkiewicz et al. (NA61 Collaboration), Nucl. Phys. A 907, 35 (2017)

[16] T. Anticic et al. (NA49 Collaboration), Phys. Rev. C 81, 064507 (2010)

[17] T. Anticic et al. (NA49 Collaboration), Eur. Phys. J. C 75(12), 587 (2015)

[18] N. G. Antoniou et al., Phys. Rev. Lett. 97, 032002 (2006)

[19] A. Bialas, and R. Peschanski, Nucl. Phys. B273, 703 (1986)

[20] F. K. Diakonos et al., PoS CPOD 2016, 010 (2017)

[21] N. Davis (NA61 Collaboration), to appear in PoS CPOD 2018 